\title{
Random Models and the Maslov Class
}

\section{Citation}

Goldfarb, Warren. 1989. Random models and the Maslov class. Journal of Symbolic Logic 54, no. 2: 460-466.

\section{Published Version}

http://dx.doi.org/10.2307/2274860

\section{Permanent link}

http://nrs.harvard.edu/urn-3:HUL.InstRepos:3200607

\section{Terms of Use}

This article was downloaded from Harvard University's DASH repository, and is made available under the terms and conditions applicable to Other Posted Material, as set forth at http:// nrs.harvard.edu/urn-3:HUL.InstRepos:dash.current.terms-of-use\#LAA

\section{Share Your Story}

The Harvard community has made this article openly available.

Please share how this access benefits you. Submit a story.

Accessibility 


\title{
RANDOM MODELS AND THE MASLOV CLASS
}

\author{
WARREN GOLDFARB
}

In [GS] Gurevich and Shelah introduce a novel method for proving that every satisfiable formula in the Gödel class has a finite model (the Gödel class is the class of prenex formulas of pure quantification theory with prefixes $\forall \forall \exists \cdots \exists$ ). They dub their method "random models": it proceeds by delineating, given any $F$ in the Gödel class and any integer $p$, a set of structures for $F$ with universe $\{1, \ldots, p\}$ that can be treated as a finite probability space $S$. They then show how to calculate an upper bound on the probability that a structure chosen at random from $S$ makes $F$ false; from this bound they are able to infer that if $p$ is sufficiently large, that probability will be less than one, so that there will exist a structure in $S$ that is a model for $F$. The Gurevich-Shelah proof is somewhat simpler than those known heretofore. In particular, there is no need for the combinatorial partitionings of finite universes that play a central role in the earlier proofs (see [G] and [DG, p. 86]). To be sure, Gurevich and Shelah obtain a larger bound on the size of the finite models, but this is relatively unimportant, since searching for finite models is not the most efficient method to decide satisfiability.

Gurevich and Shelah note that the random model method can be used to treat the Gödel class extended by initial existential quantifiers, that is, the prefix-class $\exists \cdots \exists \forall \forall \exists \cdots \exists$; but they do not investigate further its range of applicability to syntactically specified classes. In fact, a reasonably straightforward generalization of the method suffices for the Skolem classes of [DG, Chapter 3], which are classes of prenex formulas with prefixes $\forall \cdots \forall \exists \cdots \exists$ and matrices restricted in the sets of variables that may appear together in atomic subformulas.

The aim of this paper is to present a less straightforward extension of the random model method, to the Maslov class. To describe this class, we define a signed atomic formula to be an atomic formula or the negation of one, a binary disjunction to be a disjunction $(A \vee B)$ of two signed atomic formulas, and a Krom formula to be a conjunction of binary disjunctions. The Maslov class is the class of prenex formulas with prefixes $\forall \cdots \forall \exists \cdots \exists$ and matrices that are Krom formulas. The Maslov class was first shown decidable by Maslov [M]; that every satisfiable Maslov formula has a finite model was first shown by Aanderaa and the author [AG]. The latter

Received November 24, 1987.

(C) 1989, Association for Symbolic Logic $0022-4812 / 89 / 5402-0011 / \$ 01.70$ 
proof, and the somewhat different one in [DG, Chapter 4, §2], are rather complex. Here the random models method provides an impressive gain in perspicuity and brevity.

The extension of Gurevich and Shelah's method to Maslov formulas involves a key new feature. Their probability measure assigns equal probability to each structure in the space $S$; thus the probability of a set of structures is simply the ratio of its cardinality to the cardinality of $S$. At bottom, then, their proof is a counting argument: a bound is calculated on the number of structures in $S$ that make the given formula false; if the size of the structures is sufficiently large, this bound is less than the number of structures in $S$. In the proof below, however, not all structures in the space receive the same probability. In this way, the argument involves a more intrinsic use of probability.

The proof below splits into two parts. In $\S 1$ we present the logical features of Maslov formulas that are needed; in $\$ 2$ we describe the probability space and calculate the relevant bounds on probabilities.

§1. Let $F=\forall x_{1} \cdots \forall x_{m} \exists x_{m+1} \cdots \exists x_{m+n} G\left(x_{1}, \ldots, x_{m+n}\right)$ be a satisfiable Maslov formula, $m, n \geq 1$. We assume that the predicate letters in $F$ are at most $m$-adic; otherwise, dummy universal quantifiers can be added. We allow $F$ to contain 0 -adic predicate letters, that is, sentence letters. In what follows, by "atomic formula" we shall mean only those that contain a predicate letter of $F$. We assume that $F$ has the following projection property:

For each $i, 1 \leq i \leq m$, and each signed atomic formula $D$ containing exactly the variables $x_{1}, \ldots, x_{i}$, there exists an atomic formula $C$ containing the variables $x_{1}, \ldots, x_{i-1}$ such that $F$ logically implies the universal closure of $\left(C \equiv \exists x_{i} D\right)$.

Any Maslov formula can be transformed into one possessing the projection property by appropriate additions to the matrix. A signed atomic formula $D$ containing $x_{1}, \ldots, x_{i}$ is treated by introducing a new $(i-1)$-adic predicate letter $P_{D}$, conjoining $\left(\sim D \vee P_{D} x_{1} \cdots x_{i-1}\right) \cdot\left(\sim P_{D} x_{1} \cdots x_{i-1} \vee \exists x_{i} D\right)$ to the matrix, relabelling the existentially quantified $x_{i}$ as a new variable, and prenexing. Any model for the resulting formula is a model for the original one; conversely, any model for the original becomes a model for the new formula once $P_{D}$ is interpreted as an appropriate projection of the extension of the predicate letter in $D$ or the complement of that extension. (Cf. [DG, p. 117].)

A $p$-structure is a structure for the language of $F$ whose universe is $\{1, \ldots, p\}$; a $p$ atom is an atomic formula containing a predicate letter of $F$ and arguments from $\{1, \ldots, p\}$ (we ignore the distinction between the elements $1, \ldots, p$ and names for those elements). If $J$ is a truth-functional combination of $p$-atoms and $v$ is a mapping defined on all elements occurring in $J$, then $v J$ is the result of replacing each argument of each $p$-atom in $J$ by its image under $v$.

Suppose $\mathbf{N}_{0}$ is a model for $F$, with universe $N_{0}$. Let $\Phi$ be the conjunction of binary disjunctions $(C \vee D)$ whose universal closures are true in $\mathbf{N}_{0}$, where $C$ and $D$ are signed atomic formulas containing variables among $x_{1}, \ldots, x_{2 m}$; and let $\Phi_{p}$ be the conjunction of instances of $\Phi$ over the universe $\{1, \ldots, p\}$. Thus $\Phi_{p}$ is the conjunction 
of all binary disjunctions $(A \vee B)$ of signed $p$-atoms such that $\mathbf{N}_{0} \vDash v(A \vee B)$ for every mapping $v$ from $\{1, \ldots, p\}$ to $N_{0}$. The conjunction $\Phi_{p}$ is the only information we need to extract from $\mathbf{N}_{0}$ for the construction of a finite model.

(Incidentally, $\Phi$ has the following property, which is central to the solvability proofs for the Maslov class of [M] and [DG, Chapter 4, §1]. Let $\mathbf{M}$ be any model for the universal closure of $\Phi$. Then there is an extension $\mathbf{M}^{\prime}$ of $\mathbf{M}$ in which the universal closure of $\Phi$ remains true and such that $\mathbf{M}^{\prime} \vDash \exists x_{m+1} \cdots \exists x_{n} G\left[a_{1} \cdots a_{m}\right]$ for all $a_{1}, \ldots, a_{m}$ in $\mathbf{M}$. Iteration of the extension process yields a model for $F$. The proof below exploits a related property, given in Lemma 4.)

Let $J$ be a truth-functional compound of $p$-atoms. $J$ is satisfiable if $v J$ is satisfiable for some mapping $v$. For, given a truth-assignment making $v J$ true, we can obtain one making $J$ true by assigning "true" to a $p$-atom $A$ iff the given assignment assigns "true" to $v A$. In particular, then $J . \Phi_{p}$ is satisfiable provided there exists a mapping $v$ from the arguments occurring in $J$ to $N_{0}$ such that $\mathbf{N}_{0} \vDash v J$. For, given such a $v$, we may extend it arbitrarily to all of $\{1, \ldots, p\}$; then $\mathbf{N}_{0} \vDash \nu \Phi_{p}$, since $\mathbf{N}_{0} \vDash \nu \Phi_{p}$ for every mapping $v$. Thus $v J . v \Phi_{p}$ is satisfiable, since it is true in $\mathbf{N}_{0}$; whence $J . \Phi_{p}$ is satisfiable.

We now prove four fairly simple lemmas. The first gives a general property of Krom formulas, and the second gives a consequence of the projection property. The third, a corollary of the first two, is a crucial underpinning to the construction of the desired probability space. The fourth exploits the condition that the matrix of $F$ is Krom.

LEMma 1. Let $H$ be a conjunction of signed atomic formulas and let $K$ be a Krom formula. If $H . K$ is unsatisfiable, then there exist signed atomic formulas $A$ and $B$ that are conjuncts of $H$ such that A.B.K is unsatisfiable.

Proof. A truth-functional argument, using a simple induction on the number of conjuncts of the Krom formula $K$, suffices. See [AG, p. 513] or [DG, p. 109].

Lemma 2. Suppose $A$ and $B$ are signed p-atoms such that $A . B . \Phi_{p}$ is unsatisfiable. Then there is a p-atom $A^{\prime}$, whose arguments are common to $A$ and $B$, such that $A . \sim A^{\prime} . \Phi_{p}$ and $B . A^{\prime} . \Phi_{p}$ are both unsatisfiable.

Proof. If all arguments of $A$ are arguments of $B$, we may take $A^{\prime}$ to be $A$ itself. Otherwise, there exist $i$ and $j, 0 \leq i<j \leq m$, and a signed atomic formula $D$ containing the variables $x_{1}, \ldots, x_{j}$ such that $A=D\left[q_{1}, \ldots, q_{j}\right]$, where $q_{1}, \ldots, q_{j}$ are distinct elements of $\{1, \ldots, p\}$, and $q_{1}, \ldots, q_{i}$ are arguments of $B$ but $q_{i+1}, \ldots, q_{j}$ are not. By iteration of the projection property, there exists an atomic formula $C$ containing just the variables $x_{1}, \ldots, x_{i}$ such that $F$ logically implies the universal closure of $\left(C \equiv \exists x_{i+1} \cdots \exists x_{j} D\right)$. Hence $(\sim D \vee C)$ is in true in $\mathbf{N}_{0}$ for all values of $x_{1}, \ldots, x_{j}$. Let $A^{\prime}=C\left[q_{1}, \ldots, q_{i}\right]$; it follows that $\left(\sim A \vee A^{\prime}\right)$ is a conjunct of $\Phi_{p}$, so that $A . \sim A^{\prime} . \Phi_{p}$ is unsatisfiable.

Suppose $B . A^{\prime} . \Phi_{p}$ satisfiable. Then $\left(\sim B \vee \sim A^{\prime}\right)$ cannot be a conjuct of $\Phi_{p}$. Hence there is a mapping $v$ from the arguments of $B$ (which include $q_{1}, \ldots, q_{i}$ ) to $N_{0}$ such that $\mathbf{N}_{0} \vDash v B . v A^{\prime}$. Note that $v A^{\prime}$ is $C\left[v q_{1}, \ldots, v q_{i}\right]$. Since $\left(C \supset \exists x_{i+1} \ldots \exists x_{j} D\right)$ is true in $\mathbf{N}_{0}$ for all values of $x_{1}, \ldots, x_{i}$, there exist $e_{i+1}, \ldots, e_{j}$ in $N_{0}$ such that $\mathbf{N}_{0} \vDash$ $D\left[v q_{1}, \ldots, v q_{i}, e_{i+1} \cdots e_{j}\right]$. If $v$ is extended by setting $v q_{k}=e_{k}$ for $i<k \leq j$, we have $\mathbf{N}_{0} \vDash D\left[v q_{1}, \ldots, v q_{j}\right]$, that is, $\mathbf{N}_{0} \vDash v A$. Thus $\mathbf{N}_{0} \vDash v A . v B$, which contradicts the hypothesis that $A . B . \Phi_{p}$ is unsatisfiable. 
If $\mathbf{M}$ is a $p$-structure and $S \subseteq\{1, \ldots, p\}$, then let $(\mathbf{M} \mid S)$ be the conjunction of signed $p$-atoms $A$ whose arguments are in $S$ such that $\mathbf{M} \vDash A$. (In essence, $(\mathbf{M} \mid S)$ is the diagram of the members of $S$ in the structure $\mathbf{M}$.)

LEMMA 3. Let $\mathbf{M}$ be a p-structure; let $S_{1}, \ldots, S_{k}$ be subsets of $\{1, \ldots, p\}$ such that $\left(\mathbf{M} \mid S_{i}\right) . \Phi_{p}$ is satisfiable for each $i$. Then $\left(\mathbf{M} \mid S_{1}\right) \cdots\left(\mathbf{M} \mid S_{k}\right) . \Phi_{p}$ is satisfiable.

ProOF. Suppose not. By Lemma 1 there exist signed $p$-atoms $A$ and $B$ such that $A$ is a conjunct of some $\left(\mathbf{M} \mid S_{i}\right), B$ is a conjunct of some $\left(\mathbf{M} \mid S_{j}\right)$, and $A . B . \Phi_{p}$ is unsatisfiable. By Lemma 2 there exists a $p$-atom $A^{\prime}$ whose arguments are common to $A$ and $B$ such that $A . \sim A^{\prime} . \Phi_{p}$ and $B . A^{\prime} . \Phi_{p}$ are both unsatisfiable. If $\mathbf{M} \vDash \sim A^{\prime}$, then $A$ and $\sim A^{\prime}$ are both conjuncts of $\left(\mathbf{M} \mid S_{i}\right)$, so that $\left(\mathbf{M} \mid S_{i}\right) . \Phi_{p}$ is unsatisfiable; but if $\mathbf{M} \vDash A^{\prime}$ then $B$ and $A^{\prime}$ are both conjuncts of $\left(\mathbf{M} \mid S_{j}\right)$, so that $\left(\mathbf{M} \mid S_{j}\right) . \Phi_{p}$ is unsatisfiable. In either case the hypothesis is contradicted.

LEMMA 4. Suppose $\mathbf{M}$ is a p-structure in which $\Phi_{p}$ is true. Let $q_{1}, \ldots, q_{m} \in\{1, \ldots p\}$, and let $q_{m+1}, \ldots, q_{m+n}$ be distinct members of $\{1, \ldots, p\}-\left\{q_{1}, \ldots, q_{m}\right\}$. Then $\left(\mathbf{M} \mid\left\{q_{1}, \ldots, q_{m}\right\}\right) . G\left[q_{1}, \ldots, q_{m+n}\right] . \Phi_{p}$ is satisfiable.

ProOF. By Lemma 1, it suffices to show that for all signed $p$-atoms $A$ and $B$ that are conjuncts of $\left(\mathbf{M} \mid\left\{q_{1}, \ldots, q_{m}\right\}\right), A . B . G\left[q_{1}, \ldots, q_{m+n}\right] . \Phi_{p}$ is satisfiable. Since $\mathbf{M}$ makes both $\Phi_{p}$ and $A . B$ true, $(\sim A \vee \sim B)$ cannot be a conjunct of $\Phi_{p}$. Hence there is a $v$ such that $\mathbf{N}_{0} \vDash v(A . B)$. We may assume $v$ defined only on the $q_{i}$ that occur in $A . B$. Extend $v$ arbitrarily to the rest of $q_{1}, \ldots, q_{m}$. Since $\mathbf{N}_{0} \models F$, there exist members $e_{m+1}, \ldots, e_{m+n}$ of $N_{0}$ such that $\mathbf{N}_{0} \vDash G\left[v q_{1}, \ldots, v q_{m}, e_{m+1}, \ldots, e_{m+n}\right]$. Extend $v$ by setting $v q_{m+i}=e_{m+i}$ for $1 \leq i \leq n$. Then $\mathbf{N}_{0} \vDash v\left(A . B . G\left[q_{1}, \ldots, q_{m+n}\right]\right)$, which yields the desired conclusion.

\$2. Let $p>m+n$ be fixed, and let $T_{p}$ be the set of $p$-structures in which $\Phi_{p}$ is true. Lemma 3 allows us to treat an arbitrary member $\mathbf{M}$ of $T_{p}$ as generated by successive choices of truth-value assignments to $p$-atoms, as follows:

Step 0 . Arbitrarily pick values for the sentence letters in such a way that $(\mathbf{M} \mid \varnothing) . \Phi_{p}$ is satisfiable.

Step $i, 0<i \leq m$. Suppose truth-values have been chosen for all $p$-atoms containing at most $i-1$ distinct arguments in such a way that $(\mathbf{M} \mid U) . \Phi_{p}$ is satisfiable for each $U \subseteq\{1, \ldots, p\}$ of cardinality $i-1$. For each $S \subseteq\{1, \ldots, p\}$ of cardinality $i$, proceed thus. Let $\Delta$ be the set of possible values $H$ of $(\mathbf{M} \mid S)$ such that

(a) $H$ extends $(\mathbf{M} \mid U)$ for each $U \subseteq S$ of cardinality $i-1$, and

(b) $H . \Phi_{p}$ is satisfiable.

$\Delta$ is not empty, for Lemma 3 and the induction hypothesis yield the satisfiability of the conjunction of $\Phi_{p}$ with all $(\mathbf{M} \mid U)$ for $U \subseteq S$ of cardinality $i-1$. Now pick at random an $H \in \Delta$ and then fix the truth-values of the $p$-atoms whose arguments are precisely the $i$ members of $S$ in such a way that $(\mathbf{M} \mid S)=H$.

This manner of describing the determination of a random element of $T_{p}$ is but a picturesque manner of stipulating a probability measure on $T_{p}$. This measure is not such that $\operatorname{Prob}\left[\mathbf{M}=\mathbf{M}_{1}\right]=\operatorname{Prob}\left[\mathbf{M}=\mathbf{M}_{2}\right]$ for all $\mathbf{M}_{1}$ and $\mathbf{M}_{2}$ in $T_{p}$; for at various stages in the process of obtaining $\mathbf{M}_{1}$ and $\mathbf{M}_{2}$ there may have been different numbers of alternative choices available. To describe the measure precisely, call two members of $T_{p} i$-equivalent, where $0 \leq i \leq m$, iff they agree on all $p$-atoms that contain at most 
$i$ distinct arguments. For $\mathbf{M}$ in $T_{p}$, let $\#(0, \mathbf{M})$ be the number of 0 -equivalence classes, and let $\#(i+1, \mathbf{M})$ be the number of different $(i+1)$-equivalence classes into which the $i$-equivalence class of $\mathbf{M}$ splits. Finally, for each $\mathbf{M}_{1}$ in $T_{p}$, let $\operatorname{Prob}\left[\mathbf{M}=\mathbf{M}_{1}\right]$ be $\left(\prod_{0 \leq i \leq m} \#\left(i, \mathbf{M}_{1}\right)\right)^{-1}$. That is, that a structure chosen at random lie in one 0 -equivalence class is equiprobable with its lying in another; given that a structure is in a particular $i$-equivalence class, the probabilities that it lie in the $(i+1)$-equivalence classes into which the particular $i$-equivalence class splits are all equal.

We now seek to estimate the probability of the event $\mathbf{M} \vDash F$.

Estimation Lemma. There exists a $\delta, 0<\delta<1$, that does not depend on $p$ such that, for any $q_{1}, \ldots, q_{m}$ in $\{1, \ldots, p\}$,

$$
\operatorname{Prob}\left[\mathbf{M} \vDash \sim \exists x_{m+1} \cdots \exists x_{m+n} G\left[q_{1}, \ldots, q_{m}\right]\right] \leq(1-\delta)^{s}
$$

where $s=[(p-m) / n]$.

Proof. Let $q_{1}, \ldots, q_{m} \in\{1, \ldots, p\}$. Let $q_{m+1}, \ldots, q_{m+n}$ be the earliest distinct members of $\{1, \ldots, p\}-\left\{q_{1}, \ldots, q_{m}\right\}$. For each $\mathbf{M}$ in $T_{p}$ let $\psi \mathbf{M}$ be a member of $T_{p}$ such that $\psi \mathbf{M} \models G\left[q_{1}, \ldots, q_{m+n}\right]$ and $\left(\mathbf{M} \mid\left\{q_{1}, \ldots, q_{m}\right\}\right)=\left(\psi \mathbf{M} \mid\left\{q_{1}, \ldots, q_{m}\right\}\right)$. Such a $\psi \mathbf{M}$ always exists, since, by Lemma $4,\left(\mathbf{M} \mid\left\{q_{1}, \ldots, q_{m}\right\}\right) \cdot G\left[q_{1}, \ldots, q_{m+n}\right] . \Phi_{p}$ is satisfiable. Now let $\mathbf{r}=\left\langle r_{1}, \ldots, r_{n}\right\rangle$ be any $n$-tuple of distinct members of $\{1, \ldots, p\}-$ $\left\{q_{1}, \ldots, q_{m}\right\}$; let $v$ map $q_{1}, \ldots, q_{m}$ to themselves and map each $r_{i}$ to $q_{m+i}, 1 \leq i \leq n$. For any $S \subseteq\left\{q_{1}, \ldots, q_{m}, r_{1}, \ldots, r_{n}\right\}$ let $v S=\{v e \mid e \in S\}$. We wish first to estimate the probability of the event

(*) for every $p$-atom $A$ with arguments among $\left\{q_{1}, \ldots, q_{m}, r_{1}, \ldots, r_{n}\right\}$,

$$
\mathbf{M} \vDash A \quad \text { iff } \quad \psi \mathbf{M} \vDash v A .
$$

Note that, since $\psi \mathbf{M} \vDash G\left[q_{1}, \ldots, q_{m+n}\right],(*)$ implies $\mathbf{M} \vDash G\left[q_{1}, \ldots, q_{m}, r_{1}, \ldots, r_{n}\right]$, so that $\mathbf{M} \vDash \exists x_{m+1} \cdots \exists x_{m+n} G\left[q_{1}, \ldots, q_{m}\right]$. Now (*) is equivalent to the event that, for every $S \subseteq\left\{q_{1}, \ldots, q_{m}, r_{1}, \ldots, r_{k}\right\}$ and every signed $p$-atom $A$ with arguments in $S, A$ is a conjunct of $(\mathbf{M} \mid S)$ iff $v A$ is a conjunct of $(\psi \mathbf{M} \mid v S)$; in other words, for every such $S$, $(\mathbf{M} \mid S)=v^{-1}(\psi \mathbf{M} \mid v S)$. To estimate the probability, we break the event down: let $E_{0}(\mathbf{M}, \mathbf{r})$ be the event $(\mathbf{M} \mid \varnothing)=(\psi \mathbf{M} \mid \varnothing)$ and for $1 \leq i \leq m$ let $E_{i}(\mathbf{M}, \mathbf{r})$ be the event: $(\mathbf{M} \mid S)=v^{-1}(\psi \mathbf{M} \mid v S)$ for every $S \subseteq\left\{q_{1}, \ldots, q_{m}, r_{1}, \ldots, r_{n}\right\}$ of cardinality $\leq i$. By the choice of $\psi \mathbf{M}, E_{0}(\mathbf{M}, \mathbf{r})$ has probability 1 . For each $i, 1 \leq i \leq m$, let $\beta_{i}$ be the number of different atomic formulas containing the variables $x_{1}, \ldots, x_{i}$, and let $\alpha_{i}=2^{\beta_{i}}$. Then for $\mathbf{M}$ a $p$-structure and $S \subseteq\{1, \ldots, p\}$ of cardinality $i, \alpha_{i}$ is an upper bound on the number of possible values $H$ of $(\mathbf{M} \mid S)$ that extend given values of $(\mathbf{M} \mid U)$ for all $U \subseteq S$ of cardinality $i-1$. Finally, for $1 \leq i \leq m$ let $c_{i}=\left(\begin{array}{c}m+n \\ i\end{array}\right)-\left(\begin{array}{c}m \\ i\end{array}\right) ; c_{i}$ is an upper bound on the number of subsets of $\left\{q_{1}, \ldots, q_{m}, r_{1}, \ldots, r_{n}\right\}$ that have cardinality $i$ and that contain at least one $r_{k}$ (indeed, $c_{i}$ is exactly that number, unless $i \geq 2$ and $q_{1}, \ldots, q_{m}$ are not all distinct).

Now suppose $1 \leq i \leq m$ and $E_{i-1}(\mathbf{M}, \mathbf{r})$ occurs. Since $\left(\mathbf{M} \mid\left\{q_{1}, \ldots, q_{m}\right\}\right)=$ $\left(\psi \mathbf{M} \mid\left\{q_{1}, \ldots, q_{m}\right\}\right),(\mathbf{M} \mid S)=v^{-1}(\psi \mathbf{M} \mid v S)$ for each $S \subseteq\left\{q_{1}, \ldots, q_{m}\right\}$ of cardinality $i$. Hence $E_{i}(\mathbf{M}, \mathbf{r})$ occurs if correct choices are made for all $(\mathbf{M} \mid S)$ with $S \subseteq$ $\left\{q_{1}, \ldots, q_{m}, r_{1}, \ldots, r_{n}\right\}$ of cardinality $i$ that contain at least one $r_{k}$. Let $S$ be any such set. Let $\Delta$ be the set of available alternatives for $(\mathbf{M} \mid S)$, that is, the set of possible 
values $H$ for $(\mathbf{M} \mid S)$ such that $H$ extends $(\mathbf{M} \mid U)$ for each $U \subseteq S$ of cardinality $i-1$ and $H . \Phi_{p}$ is satisfiable. Then $|\Delta| \leq \alpha_{i}$. Moreover, $v^{-1}(\psi \mathbf{M} \mid v S)$ is in $\Delta$. First, since $E_{i-1}(\mathbf{M}, \mathbf{r})$ occurs, $v^{-1}(\psi \mathbf{M} \mid v S)$ extends $(\mathbf{M} \mid U)$ for each $U \subseteq S$ of cardinality $i-1$. Second, $v^{-1}(\psi \mathbf{M} \mid v S) . \Phi_{p}$ is satisfiable: for $(\psi \mathbf{M} \mid v S) . \Phi_{p}$ is satisfiable, and, if $v$ is extended arbitrarily to all of $\{1, \ldots, p\}$,

$$
v\left(v^{-1}(\psi \mathbf{M} \mid v S) . \Phi_{p}\right)=(\psi \mathbf{M} \mid v S) . \Phi_{p}
$$

$\left(\Phi_{p}\right.$ is invariant under any mapping from $\{1, \ldots, p\}$ to $\{1, \ldots, p\}$ ).

Thus, $\operatorname{Prob}\left[(\mathbf{M} \mid S)=v^{-1}(\psi \mathbf{M} \mid v S) / E_{i-1}(\mathbf{M}, \mathbf{r})\right] \geq \alpha_{i}^{-1}$; whence

$$
\operatorname{Prob}\left[E_{i}(\mathbf{M}, \mathbf{r}) / E_{i-1}(\mathbf{M}, \mathbf{r})\right] \geq \alpha_{i}^{-c_{i}} \text {. }
$$

It follows that $\operatorname{Prob}\left[E_{m}(\mathbf{M}, \mathbf{r})\right] \geq \prod_{1 \leq i \leq m}\left(\alpha_{i}^{-c_{i}}\right)$. Let $\delta$ be this product; obviously $\delta$ does not depend on $p$, and $\operatorname{Prob}\left[\operatorname{not} E_{m}(\mathbf{M}, \mathbf{r})\right] \leq(1-\delta)$.

Now since $s=[(p-m) / n]$, there exists for each $j, 1 \leq j \leq s$, an $n$-tuple $\mathbf{r}^{j}=$ $\left\langle r_{1}^{j}, \ldots, r_{n}^{j}\right\rangle$ of distinct members of $\{1, \ldots, p\}-\left\{q_{1}, \ldots, q_{m}\right\}$, such that $\mathbf{r}^{j}$ and $\mathbf{r}^{k}$ have no members in common when $j \neq k$. As we have just seen, for each $j, 1 \leq j \leq s$, $\operatorname{Prob}\left[\right.$ not $\left.E_{m}\left(\mathbf{M}, \mathbf{r}^{j}\right)\right] \leq(1-\delta)$. Moreover, the events [not $\left.E_{m}\left(\mathbf{M}, \mathbf{r}^{j}\right)\right]$ and [not $\left.E_{m}\left(\mathbf{M}, \mathbf{r}^{k}\right)\right]$ are independent if $j \neq k$, since then $\mathbf{r}^{j}$ and $\mathbf{r}^{k}$ are disjoint and whether or not $E_{m}\left(\mathbf{M}, \mathbf{r}^{j}\right)$ occurs depends only on choices made for $(\mathbf{M} \mid S)$ where $S$ is a subset of $\left\{q_{1}, \ldots, q_{m}, r_{1}^{j}, \ldots, r_{n}^{j}\right\}$ that contains at least one of $r_{1}^{j}, \ldots, r_{n}^{j}$. Hence

$$
\operatorname{Prob}\left[E_{m}\left(\mathbf{M}, \mathbf{r}^{j}\right) \text { for no } j, 1 \leq j \leq s\right] \leq(1-\delta)^{s} \text {. }
$$

Now, as noted above, if $E\left(\mathbf{M}, \mathbf{r}^{j}\right)$ then $\mathbf{M} \models \exists x_{m+1} \cdots \exists x_{m+n} G\left[q_{1}, \ldots, q_{m}\right]$. Hence, if $\mathbf{M} \models \sim \exists x_{m+1} \cdots \exists x_{m+n} G\left[q_{1}, \ldots, q_{m}\right]$ then $E_{m}\left(\mathbf{M}, \mathbf{r}^{j}\right)$ for no $j, 1 \leq j \leq s$. It follows that

$$
\operatorname{Prob}\left[\mathbf{M} \vDash \sim \exists x_{m+1} \cdots \exists x_{m+n} G\left[q_{1}, \ldots, q_{m}\right]\right] \leq(1-\delta)^{s}
$$

as desired.

COROLlARY. For large enough $p$, there exists $\mathbf{M}$ in $T_{p}$ such that $\mathbf{M} \vDash F$.

Proof. $\mathbf{M} \vDash \sim F$ iff there exists $q_{1}, \ldots, q_{m}$ in $\{1, \ldots, p\}$ such that $\mathbf{M} \vDash \sim \exists x_{1} \ldots$ $\exists x_{m+n} G\left[q_{1}, \ldots, q_{m}\right]$. By the estimation lemma, for each $m$-tuple $\left\langle q_{1}, \ldots, q_{m}\right\rangle$

$$
\operatorname{Prob}\left[\mathbf{M} \vDash \sim \exists x_{1} \cdots \exists x_{m+n} G\left(q_{1}, \ldots, q_{m}\right)\right] \leq(1-\delta)^{s} .
$$

Since there are $p^{m} m$-tuples $\left\langle q_{1}, \ldots, q_{m}\right\rangle$ of elements of $\{1, \ldots, p\}$,

$$
\operatorname{Prob}[\mathbf{M} \models \sim F] \leq p^{m}(1-\delta)^{s} .
$$

Since $s=[(p-m) / n]$, for large enough $p$, Prob $[\mathbf{M} \models \sim F]<1$. Hence, for large enough $p$ there exists a member of $T_{p}$ that makes $F$ true.

Let $\alpha=\prod_{1 \leq i \leq m}\left(\alpha_{i}^{c_{i}}\right)$, that is, $\delta^{-1}$. Since $\alpha$ is much bigger than $m$ and $n$, if $p \geq \alpha(\log \alpha)^{2}$ then $p$ is "large enough" that $\operatorname{Prob}[\mathbf{M} \vDash \sim F]<1$. This bound on the size of the smallest finite model of $F$ is somewhat better than those obtained from the proofs in [AG] or [DG].

Acknowledgements. I am most grateful to George Boolos and Burton Dreben for their helpful suggestions. 


\section{REFERENCES}

[AG]' S. AanderaA and W. Goldfarb, The finite controllability of the Maslov class, this Journal, vol. 39 (1974), pp. 509-518.

[DG] B. DREBEN and W. GoldFarb, The decision problem: Solvable classes of quantificational formulas, Addison-Wesley, Reading, Massachusetts, 1979.

$[\rightarrow$ K. GöDEL, Zur Entscheidungsproblem des logischen Funktionenkalküls, Monatshefte für Mathematik und Physik, vol. 40 (1933), pp. 433-443.

[GS] Y. GUREVICH and S. SHELAH, Random models and the Gödel case of the decision problem, this JOURNAL, vol. 48 (1983), pp. 1120-1124.

[M] S. MASLov, An inverse method of establishing deducibilities in the classical predicate calculus, Doklady Akademii Nauk SSSR, vol. 159 (1964), pp. 17-20; English translation, Soviet Mathematics Doklady, vol. 5 (1964), pp. 1420-1424.

DEPARTMENT OF PHILOSOPHY

HARVARD UNIVERSITY

CAMBRIDGE, MASSACHUSETTS 02138 